\title{
BMJ Open Factors contributing to medicine-related problems in adult patients with diabetes and/or cardiovascular diseases in Saudi Arabia: a qualitative study
}

Abdullah Mahdi Al Hamid, ${ }^{1}$ Maisoon Ghaleb, ${ }^{1}$ Hisham Aljadhey, ${ }^{2}$ Zoe Aslanpour ${ }^{1}$

To cite: Al Hamid AM, Ghaleb M, Aljadhey $\mathrm{H}$, et al. Factors contributing to medicine-related problems in adult patients with diabetes and/or cardiovascular diseases in Saudi Arabia: a qualitative study. BMJ Open 2017;0:e017664. doi:10.1136/ bmjopen-2017-017664

- Prepublication history and additional material for this paper are available online. To view these files, please visit the journal online (http://dx.doi. org/10.1136/bmjopen-2017017664).

Received 8 May 2017 Revised 9 August 2017 Accepted 16 August 2017

\section{CrossMark}

${ }^{1}$ Department of Pharmacy, Pharmacology and Postgraduate Medicine, School of Life and Medical Sciences, University of Hertfordshire, Hatfield, Hertfordshire, UK

${ }^{2}$ College of Pharmacy, King Saud University, Riyadh, Riyadh, Saudi Arabia

Correspondence to Dr Abdullah Mahdi Al Hamid; a.alhamid@herts.ac.uk

\section{ABSTRACT}

Objectives To investigate the factors contributing to medicine-related problems (MRPs) among patients with cardiovascular diseases (CVDs) and/or diabetes in Saudi Arabia.

Design Qualitative semistructured interviews were conducted. Interviews were audio recorded then transcribed into Microsoft Word. The transcribed interviews were then imported into the qualitative analysis software NVivo where thematic analysis was applied. Thematic synthesis was achieved by coding and developing subthemes/themes from the findings of the interviews. Setting Five healthcare centres in Najran, Saudi Arabia. Participants 25 adult patients with diabetes and/or CVDs. Results The study cohort included 16 men and 9 women with a median age of 61.8 years (40-85 years). Diabetes was the main condition encountered among 23 patients and CVDs were reported among 18 patients. Perceived factors leading to MRPs were of four types and related to: patient-, healthcare system-, clinical (condition-) and medicine-related factors. Patient-related factors were related to living situation, religious practices, diet/ exercise and patients' behaviour towards the condition and medicines. Healthcare system-related factors comprised sources and availability of medicines, ease of access to healthcare system and patient satisfaction with healthcare providers. Clinical (condition-) related factors associated with both the knowledge and control over condition, and effects of the condition among medicines intake. Medicine-related factors included lack of knowledge about medicines and medicine use.

Conclusions The results of this study uncovered many factors associated with MRPs among patients with CVDs and diabetes in Saudi Arabia, especially in reference to lifestyle and medicine use. Improving communication with healthcare professional alongside the introduction of national clinical guidance would mitigate the unwanted health complications related to medicine use.

\section{INTRODUCTION}

Medicine-related problem (MRP) is defined as 'an event or circumstance involving drug therapy that actually or potentially interfere with the desired outcome'. ${ }^{1}$ Research shows that MRPs contribute to a high number of
Strengths and limitations of this study

- The study explored factors related to patients behaviour and attitudes towards medicine use in diabetes and/or cardiovascular diseases.

- The study employed semistructured interviews in five different regions within Saudi Arabia, providing realistic account for other regional states in the Gulf region as they share most demographic characteristics.

- The outcomes of the study can be integrated in future interventions to tackle medicine-related problems.

- The findings of the study may not be generalised to other locations with different populations.

- There was no first-hand observational data to compare with the interview interpretations.

morbidities and mortalities worldwide as well as an increased healthcare expenditure. ${ }^{2}$ MRPs are also responsible for undesirable health consequences in patients that often result in hospitalisation. Previous published systematic reviews and meta-analyses estimated that MRPs accounted for up to $10 \%$ of total hospitalisation. ${ }^{3-5}$ MRPs can occur at any stage of the medicine use and could be affected by many factors related mainly to the: condition, patient and/or medicines. ${ }^{3}$ Patients with cardiovascular diseases (CVDs) and/or diabetes mellitus (DM) are often on polypharmacy which increases their susceptibility to MRPs. ${ }^{6-8}$

Polypharmacy has been reported as one of the major risk factors leading to MRPs in adult patients. ${ }^{9}{ }^{10}$ Additional risk factors that have been reported in the literature include: old age, female gender, depression, immobilisation, cohabitation and lack of education. ${ }^{611-15}$ However, these factors were mainly extracted from quantitative studies, which comprised either direct observations or reviewing the medical records retrospectively. Hence, a limited number of qualitative studies have 
explored factors contributing to MRPs from the patients' perspectives. ${ }^{3}$ Precisely, these studies investigated the relationship between MRPs and socioeconomic status and lifestyle ${ }^{16-28}$; knowledge regarding medicine and adherence to therapy and knowledge about the condition and its management. ${ }^{1629-35}$ From the aforementioned studies the following factors emerged: patients' behaviour towards healthcare systems/medicines/conditions. Also, patients' beliefs, family history of condition, type of diet, lack of exercise, smoking, excess alcohol intake, excess caffeine intake and stress. ${ }^{16}{ }^{29-35}$ However, it is crucial to take into consideration that these factors were extracted from studies carried out in only 12 countries, which included: Australia, ${ }^{28}$ Brazil $^{27}$ Cameroon, ${ }^{30}$ Canada, ${ }^{29}$ Croatia, ${ }^{25}$ Ireland,${ }^{24}$ Malaysia, ${ }^{31}{ }^{32}$ South Africa, ${ }^{22}$ Spain, ${ }^{32}$ Taiwan, ${ }^{34}$ the UK $\mathrm{UK}^{6} 1719212635$ and the USA. ${ }^{182023}$ Yet, to date no study has investigated factors leading to MRPs in patients with CVDs and/or DM in Saudi Arabia or even in the Middle East.

Therefore, this study aims to explore the potential contributory factors leading to MRPs in adult patients with CVDs and/or DM in Saudi Arabia.

\section{METHODS}

\section{Study design}

The study used semistructured interviews with adult patients who had diabetes and/or CVD. The main aims of the interviews were to explore the contributory factors that could lead to MRPs. The interview guide included core questions for all the participants and allowed flexibility to follow-up further information (online supplementary appendix I).

\section{Patient population}

Participants were recruited from five randomly selected healthcare centres (HCCs) located at both urban and rural areas within Najran region, Saudi Arabia. The interviews were conducted between June and July 2014 in private rooms directly after the patients' visit to the Chronic Disease Clinics in each centre. A purposive sampling approach was adopted, ${ }^{36-38}$ where patients recruited were adults aged $\geq 18$ years, who had CVDs and/ or DM (according to the ICD-10 codes; ICD, International Classification of Diseases) and gave full verbal consent for the interview. Data saturation was reached after the 21st interview. However, data collection process was continued until organised themes constructed. Patients excluded were those who could not communicate and/or did not give their consent for any reason.

\section{Participants' recruitment}

Potential eligible participants were identified from the existing databases of the HCCs through the diagnosis codes for CVDs and DM (ICD-10 codes), assigned to them when they were transferred to the HCCs to check-up and take their repeated prescriptions once monthly. Before contacting the patient to obtain informed consent, the patient's attending physician was contacted for authorisation to approach the patient. Once the physician's authorisation was obtained, a trained nurse approached each potential participant in order to confirm the study eligibility. Thereafter, eligible participants were provided with informed consent for the study. Diagnosis was confirmed for each patient after they agreed to participate in the study and gave their consent. Prior to the interviews AMAH reconfirmed the consents of patients verbally. Participants were also made aware that they could withdraw and leave the study at any time they would like to. Moreover, the objectives of the study were explained to the patients at the beginning of each interview.

\section{Data collection}

A total of 25 patients met the study inclusion criteria and were interviewed. Semistructured interviews were conducted by the researcher (AMAH) at the five HCCs (online supplementary appendix I). Each patient was interviewed independently. All interviews were audio recorded using a Sony voice recordable frequency modulation radio, with the patient's permission. In addition, the researcher took notes during the interview when required. For instance, notes were taken for non-verbal responses such as smiling, looking sad, feeling angry, among others. Each interview started with a demographic section to confirm patient's age, nationality, language, marital status, religion and education level. This was followed by open questions regarding the patients' experiences with clinical-related, lifestyle-related and medicine-related factors that may lead to MRPs. Patients were made aware that there was no right or wrong answer, and hence were encouraged to give their opinions honestly and openly. Moreover, when required, probing questions were asked to encourage the patients to express their views. At the end of each interview, patients were given the opportunity to comment and ask questions about the topics of the interview. Finally, participants were thanked for their contribution to the study and invited to listen to the recording if they wished. Each interview lasted around $30 \mathrm{~min}$.

\section{Translation}

The interviews were conducted in Arabic and the notes taken during the interviews were also in Arabic. The final transcripts of the interviews were translated into English by the researcher. To validate the translation, one of the research supervisors, who is also bilingual, translated a sample of the interviews. Then, any issue was resolved by discussion.

The translation of the interview scripts was made using a back-translation technique. In this respect, the interview scripts were translated by two translators (the researcher and one of his supervisors); then, the back-translation into English was made by a further two bilingual translators within the department. This was followed by a group discussion between the researcher and the translators, where the original version was compared with the 
translated version. Only minor differences were found, particularly in the expressions related to the local culture. Consequently, appropriate changes to the wordings were made in the original version to make it theoretically equivalent to the English version.

\section{Data analysis}

An ethnographic approach to data analysis was used in order to support the interaction between theory, resources and methods. ${ }^{39}$ This was started by reviewing the literature regarding MRPs among adult patients with CVDs and/or DM. The outcomes of the review were discussed among the researchers. As a result, the relevant outcomes were used in framing the semistructured guide ${ }^{40}$ and the following subjects were included: patient, clinical and lifestyle (online supplementary appendix II). Each interview was transcribed into Microsoft Word 2007 and labelled with each participant's unique reference code. The transcribed interviews were discussed among researchers to enhance the analysis. Then the interviews were imported into the qualitative software package NVivo beta for Mac; which was used for the coding and extraction of themes and subthemes. ${ }^{41}$ Inductive approach was adopted in order to allow themes to develop from open-ended questions. Primary coding of data reflected the problems relating to medicines, and was developed by two independent members of the research team. The results were compared and any discrepancies were resolved by discussion. Similarly, secondary coding was applied to the primary categories and provided more details regarding the patients' knowledge and attitude. The coding framework clarified what should be included in each category and ensured that the extracted themes were of significant to the topic. In order to ensure trustworthiness of the coding procedure, coding was undertaken by two members of the research team and an additional independent member who had not been involved with the development of the coding frame.

\section{RESULTS}

Twenty-five patients (of a total of 40 invited to take part) participated in the study (response rate 62.5\%) at five HCCs in Najran, Saudi Arabia. The aforementioned patients included 16 adult men and 9 women, with a median age of 61.8 years ( $40-85$ years). The majority of the patients were Saudis $(n=20)$, whereas fewer were Yemenis $(n=3)$, Egyptian $(n=1)$ and Indian $(n=1)$. All of the patients were Muslims. The marital status of the included patients was as follows: 18 were married, 1 widow, 3 divorced and 3 unknown as they preferred not to say. Regarding the educational level, 13 patients were illiterate, 3 could read and write without a certificate, 8 had a school certificate and only 1 had a bachelor's degree. All of the patients spoke Arabic and one was bilingual (Arabic and Urdu).

Patients were identified whether they had MRPs at a screening interview. In this respect, patients with MRPs were more likely to agree to be interviewed. From the participant experience, four groups of risk factors leading to MRPs and included: patient-, healthcare system-, clinical (condition-) and medicine-related factors (table 1).

\section{Patient-related factors \\ Sociodemographic factors}

Regarding the patients' conditions, DM was the main condition encountered by 23 patients. CVDs were also reported in 16 patients including hypertension (HTN), hypercholesterolaemia and ischaemic heart disease. On the other hand, two patients suffered from CVDs only. Also, comorbidities were reported alongside the main conditions in 12 patients and were mainly abdominal disturbances, anaemia, neuropathy and hypercholesterolaemia.

The most common reported medicinal classes were antihypertensive and heart medicines, whereas, for DM were oral hypoglycaemic agents and insulin. Unexpectedly polypharmacy was not an issue among participants. Hence, the average number of medicines taken per patient was 3.5 (range 2-6).

Three social factors emerged from the interviews, and were: number of people living at home, help at home with medicines and religious practices interfering with medicine use and adherence to medicine regimen. None of the patients was living alone, and the mean number of people living at home with patients was seven (range 2-13). In this respect, family members often helped patients with their medicine intake as one patient responded to a question if he takes his medicine by himself:

No, my children do. I did not know how to use it myself.

They tried to teach me in the dispensary but I failed to learn.

My children are educated;

one of them is a pharmacist and gives it to me (Patient $15)$.

Only six patients reported that they use the medicines by themselves. Yet, the patients' medicine intake was affected by religious practices such as pilgrimage and fasting where patients admitted missing/delaying medicines until breaking their fasting. One patient considered pilgrimage as an alternative cure to their condition and did not take the medicines:

I never take it (medicine) during Hajj or Omrah because walking during Hajj and Omrah relieves me...

so I don't need to take it (Patient 1).

\section{Lifestyle factors}

Lifestyle factors contributing to MRPs among participants were body weight/body mass index, poor diet, smoking and lack of physical activity. A total of nine patients reported obesity or overweight; whereas, 11 did not know their body weight. On the other hand, only five patients 
Table 1 Themes and subthemes emerging from the interviews

\begin{tabular}{|c|c|}
\hline Category & Themes/subthemes identified within this category \\
\hline Patient-related factors & $\begin{array}{l}\text { Sociodemographic factors } \\
\text { Demographic factors } \\
\text { Overweight/obesity } \\
\text { Blood glucose level } \\
\text { Blood pressure } \\
\text { Smoking } \\
\text { Social factors } \\
\text { Number of people at home } \\
\text { Help at home with medicines } \\
\text { Religious practices such as fasting and pilgrimage interfering with taking medicines } \\
\text { Lifestyle-related factors } \\
\text { Physical activity } \\
\text { Patients lifestyle in relation to exercise } \\
\text { Daily exercise } \\
\text { Lack of time for exercise } \\
\text { Lack of facilities for exercise } \\
\text { No motivation towards exercise } \\
\text { Perception of daily activities as exercise } \\
\text { Diecial/cultural restrictions } \\
\text { Regular/irregular meals } \\
\text { Overeating due to food craving or social gatherings } \\
\text { Awareness of diet importance } \\
\text { Types and availability of meals } \\
\text { Smoking } \\
\text { Healthcare system-related factors } \\
\text { Healthcare system } \\
\text { Ease of access to healthcare system } \\
\text { Patient satisfaction with healthcare system } \\
\text { Availability of medicines } \\
\text { Relationship with healthcare providers } \\
\text { Education time and quality } \\
\text { Counselling and support } \\
\text { Communication in healthcare professionals } \\
\text { - instructions from healthcare providers }\end{array}$ \\
\hline Medicine-related factors & $\begin{array}{l}\text { Knowledge about medicines } \\
\text { Knowledge about medicine effects and side effects } \\
\text { Knowledge of medicine names and doses } \\
\text { Fear of side effects } \\
\text { Belief in alternative and natural therapies (fear of medicines being toxic chemicals) } \\
\text { Belief that exercise can control the condition (replace medicines) } \\
\text { Belief that diet can control the condition (replace medicines) } \\
\text { Medicine intake } \\
\text { Number of medicines } \\
\text { Ease of use of medicines } \\
\text { Difficulty in medicine intake } \\
\text { Forgetfulness to take medicines } \\
\text { Follow-up of instructions in relation to medicine intake } \\
\text { Refusal to take medicines } \\
\text { Not taking medicines because of fasting }\end{array}$ \\
\hline
\end{tabular}

CVD, cardiovascular disease; DM, diabetes mellitus. 
claimed healthy weight. Despite the reported obesity/ overweight, the majority of patients $(n=20)$ reported eating healthy and balanced diet two to three times a day. Yet, 13 of these patients were smokers or ex-smokers. When asked about their physical activities, 11 patients had misconception regarding exercises. They perceived certain activities as exercise such as walking to work, working in a car workshop/market. Additionally, three patients could not exercise due to physical incapability and four due to lack of time. In those patients, physical inability caused fluctuations in the glucose/blood pressure levels which in turn affected the effective condition control. In many of these cases doctors found it very challenging to prescribe the accurate dose and maintain it for long term.

\section{Patients' behaviour towards their condition and medicines}

Various contributory factors relating to conditions and medicines were identified mainly where patients' behaviour towards the condition and medicine intake will enable them gain more control over their conditions. Six interviewees declared adjusting their medicine doses and modalities of intake according to: condition, nutrition (food intake), blood sugar level and condition improvement (less diabetic complications). Likewise, patients acknowledged control over their conditions mainly relating to increased blood sugar level or blood pressure. Hence, patients with diabetes claimed to have control over their blood sugar level. They indicated measuring their glucose level at home (with their own devices) and adjusting it with regulating their food consumption. Yet, if the level was high, they either stayed at home and rested, or adjusted the dose of insulin/oral hypoglycaemic agent. A similar attitude was stated regarding uncontrolled blood pressure. In all cases (diabetes and HTN) patients reported that they controlled their health problems and did not report them to the healthcare system. Hence, active decision-making was an important factor in prompting adherence to medicines.

\section{Healthcare system-related factors}

Additional emerging factors that affected adherence and hence caused MRPs were related to the healthcare system, namely: the sources and availability of medicines, ease of accessibility and patient satisfaction with the healthcare providers. Governmental hospitals/dispensaries were the main source of medicines. However, in a very few cases $(n=4)$ if medicines were not available in governmental hospitals, community pharmacies were the second option. Thus, 21 patients used government dispensaries to access their medicines, whereas three patients took their medicines only from community pharmacies, and one has had his medicines delivered to his home from a private hospital. Community pharmacies were accessed only when medicines (such as multivitamins) were not available in the governmental dispensaries. Consequently, the ease of access to medicines and healthcare system was attained by a few limitations being: waiting time for prescription processing, the limited opening hours of the chronic conditions' clinic. Yet, patients did not relate the long waiting time to satisfaction with the quality of the provided healthcare services. Thus, the majority of patients $(n=21)$ were satisfied with the quality of the offered healthcare services. Only four patients complained that HCCs were crowded with patients with no predetermined appointments and the limited access times (ie, the clinic runs only once a week). They further complained that healthcare providers did not provide enough information for patients. Additionally, major concerns arose regarding lack of communication and decreased contact time with the healthcare professionals. The aforementioned concerns negatively influenced the patients' relationship with healthcare providers. Thus, the patients reported their comments on counselling and support, communication barriers, understanding the language, education quality and time and lack of trust towards the healthcare professionals:

No, they do not understand me. They never tell me anything.

They write down my medicine on a piece of paper to get them but they never explained anything. The doctor is always busy and thinks that we understand his writing (Patient 22).

Fewer patients were satisfied with the service provided by the healthcare professionals and appreciated their advice. However, some of them did not follow the given advice or did not understand them initially.

\section{Condition-related factors}

Principally, not following the healthcare professionals' advice often resulted in patients' lack of knowledge regarding their conditions which then affected their medicines' intake. When asked about their conditions, most of the patients stated that they had more experience and knowledge about their disease(s) (CVDs and DM) than healthcare professionals. Patients believed they could manage their conditions without need to the healthcare professionals:

Diabetic and blood pressure patient knows how to treat himself (Patient 21).

This was partly because of the decreased contact time and lack of communication with the healthcare professionals. Consequently, patients with both CVDs and diabetes lacked control over their conditions. Specifically, patients with diabetes had fluctuating blood glucose levels between $70 \mathrm{mg} / \mathrm{dL}$ and $350 \mathrm{mg} / \mathrm{dL}$ (normal level $\leq 126 \mathrm{mg} / \mathrm{dL}$ ). Patients attributed the fluctuation in their blood glucose level to: carelessness, the amount and type of the food they have and ineffectiveness of the provided treatment. Similarly, hypertensive patients did not show control over their fluctuating blood pressure; which they attributed to the life stress. 
'My blood pressure level goes high when I am nervous but most of the time it is fine' (Patient 16).

This poor management of the conditions led to regular changes in their medicines and doses by their doctors.

\section{Medicine-related factors}

The lack of knowledge regarding the medicines and ideal medicine use as well as the regular changing of medicine doses have led in many cases to poor management of the condition. Only one patient could identify the names and shapes of medicines/containers. Similarly, they could not tell the effects of each medicine and their knowledge was limited to the general indication of them (such as CVDs or DM). A very few patients $(\mathrm{n}=4)$ knew the side effects of their medicines. Other patients had a misconception regarding side effects of medicines. Thus, one patient believed that insulin completely destroys the body.

I totally refused to use insulin and I take tablets instead, although the doctor has always been advising me to take insulin, but one of my relatives says that it totally destroys the pancreas. With tabs, I take $500 \mathrm{mg}$ after meals and $5 \mathrm{mg}$ before breakfast and dinner' (Patient 1).

Moreover, three patients believed that diet and exercise could cure DM rather than using medicines to manage it.

Patients also relied on family members in taking medicines at certain time periods. A very few patients $(n=3)$ reported missing doses. However, during the fasting times the whole treating regimen is being changed, which often affected patients' adherence to their medicines. Patients described their adherence by expressions like: 'regularly', 'ordinarily', 'taking them as prescribed', 'everywhere' and 'all the time including when travelling'.

The variability in the medicine intake's pattern did not affect the effectiveness of the medicine regimen from the patients' perspective. Hence, most patients were satisfied with the treatment. Only few issues were encountered with patients and included: not feeling relieved, losing consciousness, appetite suppression, nausea and craving for food. This led in some cases to non-adherence to the regimen. In this respect, patients refused to take their medicines because they were unsatisfied with the treatment, felt irritated after taking medicines, being ill, being lazy, or had a preference of tablets over injection.

\section{DISCUSSION}

To the best of our knowledge, this is the first qualitative study conducted regarding to explore the potential risk factors leading MRPs in adult patients with CVDs and diabetes in Saudi Arabia from the patients' perspectives and practices. In this respect, the findings of this study have enabled richer understanding of the main risk factors relating to patient, healthcare, clinical and medicines which may lead to MRPs.
Although similar risk factors were reported in other countries, patients in this study showed divergent contributory risk factors leading to MRPs. For instance, unlike previous studies ${ }^{6910}$ the patients in this study were with multiple comorbidities but were not on polypharmacy with an average of only $3-4$ medicines. This could be attributed to the lack of national clinical guidance which have been used in the developed countries as preventive measures. ${ }^{4243}$ Other social factors that were specific to the interviewed cohort were the patients' living situation and religious practices. The patients' dependant living situation made them rely on family members to get help with medicines; and this in turns led to MRPs as highlighted in previous studies. ${ }^{21}$ Moreover, patients' religious practices including hajj and Omrah had a significant impact on delay in medicines intake and/or missing doses. This confirmed data from previous research that examined the effect of religion on MRPs among Muslim patients in the UK. ${ }^{16} 1921$

Furthermore, lifestyle risk factors including diet, exercise and smoking showed to have a negative impact on the patients' medicine use, which is consistent with the findings from other studies that investigated these issues in different parts of the world. ${ }^{16-27} 2934$ Patients showed misconception in relation to exercise; where they considered walking to work as exercise. Other patients could not exercise due to lack of time, age, stress and/or disease. Poor diet also was a more prominent issue in this study when compared with other studies in different countries. ${ }^{16-23} 2631$ Hence, patients in this study showed frustration from being overweight and expressed tiredness. Moreover, language was an issue, which made it difficult for patients to understand healthcare professionals. This was encountered in similar studies conducted with ethnic minorities in the UK who did not speak English well. ${ }^{16171921}$ The majority of the healthcare team in Saudi Arabia was composed of expatriates who did not speak or spoke little Arabic.

Subsequently, lack of communication with the healthcare teams influenced the patients' satisfaction with the healthcare system. That was shown through the patients' failure to apply the instructions and recommendations provided regarding the management of their conditions. Previous research confirmed that patients who did not understand doctors claimed that they did not get enough information regarding their disease and medicines. ${ }^{1617243135}$ This was exacerbated by the short consultation time with healthcare professionals. The absence of a national patient safety agency in Saudi Arabia that assesses the quality of the provided services could weaken the culture of accountability among healthcare professionals to follow the good practice rules.

All the aforementioned barriers led to patients' lack of control over their condition(s) and the subsequent undesirable complications. It is noteworthy that patients with CVDs and diabetes from many countries showed limited knowledge about their conditions, their nature and the symptoms associated with it ${ }^{26} 28293233$ which adversely 
affect the management process. Similarly, patients revealed limited knowledge regarding medicines' mode of action, effects and side effects despite their ability to identify the class of medicine that they are using. In most cases, patients counted on family and friends regarding these significant issues. This lack of knowledge about the condition resulted in lack of control over it and its complications. Subsequently, patients did not take their medicines as required which in many occasions resulted in treatment ineffectiveness. For instance, patients reported fluctuated blood pressure and blood sugar levels which they adjusted by changing medicine doses or food intake without referring to healthcare professionals. This could explain their high level of satisfaction with the healthcare system; but low to moderate level of satisfaction with healthcare professionals. Additional factors affecting their medicine use included: polypharmacy, religious duties (pilgrimage and fasting) and fear of side effects. The latter findings were consistent with studies conducted with Muslim population in the UK, where religious duties implicated MRPs. ${ }^{17} 1921$

\section{Strengths and weaknesses}

This study uncovered many risk factors relating to MRPs that can be incorporated into future screening tools to predict and identify MRPs. There is very little research regarding patients' views in Saudi Arabia so the study was timely in informing about patients' perspectives of factors relating MRPs. The study was limited to patients with CVDs and/or DM and may not be generalisable to patients with other long-term conditions. Due to the qualitative nature of the study, the interpretation of semistructured interviews could be subjective; hence, caution should be applied in interpretation. However, this was minimised by using the interview guide consistently during the interviewing process and validating the interview transcripts.

\section{Implication for clinicians and policy makers}

The burden of non-communicable diseases (such as CVDs and DM) has been increasing over the last few years in Saudi Arabia. Exploring factors contributing to MRPs from patients' statements assist in reducing the numbers of unnecessary/unplanned hospital admission. This study acknowledged the most important factors contributing to MRPs particularly those relating to patients' behaviour. The findings of the research have clear implications for practice and policy that are also applicable to other regional states in the Gulf region that share most of the demographic and practice characteristics.

\section{CONCLUSIONS}

The findings of this study addressed a gap in the literature relating to the risk factors leading to MRPs among patients with CVDs and diabetes in Saudi Arabia using a qualitative approach. These factors were mainly attributed to the insufficient support provided for patients through the healthcare professionals and subsequently deficiency of knowledge about the condition(s) and medicines among patients. Additionally, it is evident that an introduction of a national clinical guidance would help managing patients with CVDs and DM to achieve favourable outcomes. This guidance should take into account patients' perceptions and practices towards their condition $(\mathrm{s})$, lifestyle and medicines.

Acknowledgements The authors would like to thank the five healthcare centres for hosting the study.

Contributors AMAH carried out the interviews, data analysis and drafted the final manuscript. ZA contributed to the study design, coordination of the study and helped drafting the manuscript. HA contributed to data analysis and drafting the manuscript. MAG contributed to the study design and revised the manuscript critically for important intellectual content and edited the final draft. All authors read and approved the final manuscript. The interviews were conducted by AMAH during his $\mathrm{PhD}$ studies. Prior to conducting the interviews, AMAH received three intensive trainings on qualitative data collection, analysis and interpretation.

Disclaimer I confirm that the views expressed in the submitted article are my own and not an official position of the institution or funder.

Competing interests None declared.

Patient consent Obtained.

Ethics approval Ethical approval was sought from the General Directorate of Health Affairs in Najran, Saudi Arabia. In addition, informed consent was obtained from each patient before the interview took place. The interviewees were further provided with a study information sheet prior to the interview containing detailed information about the interview process, the use of the information obtained, as well as the researcher's commitment to maintaining patients' confidentiality. Moreover, participants were made aware before the interview that they have the right to withdraw anytime without giving any justification.

Provenance and peer review Not commissioned; externally peer reviewed.

Data sharing statement The datasets generated and/or analysed during the current study are not publicly available due to restrictions associated with anonymity of participants but are available from the corresponding author on reasonable request.

Open Access This is an Open Access article distributed in accordance with the Creative Commons Attribution Non Commercial (CC BY-NC 4.0) license, which permits others to distribute, remix, adapt, build upon this work non-commercially, and license their derivative works on different terms, provided the original work is properly cited and the use is non-commercial. See: http://creativecommons.org/ licenses/by-nc/4.0/

(C) Article author(s) (or their employer(s) unless otherwise stated in the text of the article) 2017. All rights reserved. No commercial use is permitted unless otherwise expressly granted.

\section{REFERENCES}

1. Pharmaceutical Care Network Europe. Classification of drug related problems. 2010. http://www.pcne.org/upload/files/11_PCNE_ classification_V6-2.pdf

2. NHS. The National Health Service (Pharmaceutical Services) Regulations 2005 The National Archives. (Vol 641). UK.

3. Al Hamid A, Ghaleb M, Aljadhey $\mathrm{H}$, et al. A systematic review of qualitative research on the contributory factors leading to medicine-related problems from the perspectives of adult patients with cardiovascular diseases and diabetes mellitus. BMJ Open 2014;4:e005992.

4. Howard RL, Avery AJ, Slavenburg S, et al. Which drugs cause preventable admissions to hospital? A systematic review. $\mathrm{Br} J$ Clin Pharmacol 2007;63:136-47.

5. Schneeweiss S, Hasford J, Göttler M, et al. Admissions caused by adverse drug events to internal medicine and emergency departments in hospitals: a longitudinal population-based study. Eur J Clin Pharmacol 2002;58:285-91.

6. Gordon K, Smith F, Dhillon S. Effective chronic disease management: patients' perspectives on medication-related problems. Patient Educ Couns 2007;65:407-15. 
7. Milicevic Z, Raz I, Beattie SD, et al. Natural history of cardiovascular disease in patients with diabetes: role of hyperglycemia. Diabetes Care 2008;31:S155-S160.

8. Schainberg A, Ribeiro-Oliveira A, Ribeiro JM. Is there a link between glucose levels and heart failure? An update. Arq Bras Endocrinol Metabol 2010;54:488-97.

9. Benson J, Britten N. Patients' decisions about whether or not to take antihypertensive drugs: qualitative study. BMJ 2002;325:873.

10. Cottrell WN, Denaro CP, Emmerton L. Exploring beliefs about heart failure treatment in adherent and nonadherent patients: use of the repertory grid technique. Patient Prefer Adherence 2013;7:141-50.

11. Andreazza RS, Silveira De Castro M, Sippel Köche P, et al. Causes of drug-related problems in the emergency room of a hospital in southern Brazil. Gac Sanit 2011;25:501-6.

12. Carrasco-Garrido $P$, de Andrés LA, Barrera VH, et al. Trends of adverse drug reactions related-hospitalizations in Spain (2001-2006). BMC Health Serv Res 2010;10:1.

13. Franceschi M, Scarcelli C, Niro V, et al. Prevalence, clinical features and avoidability of adverse drug reactions as cause of admission to a geriatric unit. Drug Saf 2008;31:545-56.

14. van der Hooft CS, Sturkenboom MCJM, van Grootheest K, et al. Adverse drug reaction-related hospitalisations. Drug Saf 2006;29:161-8.

15. Wawruch M, Zikavska M, Wsolova L, et al. Adverse drug reactions related to hospital admission in Slovak elderly patients. Arch Gerontol Geriatr 2009;48:186-90.

16. Brown K, Avis M, Hubbard M. Health beliefs of African-Caribbean people with type 2 diabetes: a qualitative study. Br J Gen Pract 2007;57:461-9.

17. Choudhury SM, Brophy S, Williams R. Understanding and beliefs of diabetes in the UK Bangladeshi population. Diabet Med 2009;26:636-40.

18. Coronado GD, Thompson B, Tejeda S, et al. Attitudes and beliefs among Mexican Americans about type 2 diabetes. J Health Care Poor Underserved 2004;15:576-88.

19. Grace C, Begum R, Subhani S, et al. Prevention of type 2 diabetes in British Bangladeshis: qualitative study of community, religious, and professional perspectives. BMJ 2008;337:a1931.

20. Hu J, Amirehsani K, Wallace DC, et al. Perceptions of barriers in managing diabetes: perspectives of Hispanic immigrant patients and family members. Diabetes Educ 2013;39.

21. Lawton J, Ahmad N, Hanna L, et al. I can't do any serious exercise': barriers to physical activity amongst people of Pakistani and Indian origin with Type 2 diabetes. Health Educ Res 2006;21:43-54.

22. Mshunqane N, Stewart AV, Rothberg AD. Type 2 diabetes management: Patient knowledge and health care team perceptions, South Africa. Afr J Prim Health Care Fam Med 2012;4.

23. Rustveld LO, Pavlik VN, Jibaja-Weiss ML, et al. Adherence to diabetes self-care behaviors in English- and Spanish-speaking Hispanic men. Patient Prefer Adherence 2009;3:123-30.

24. Smith SM, O'Leary M, Bury G, et al. A qualitative investigation of the views and health beliefs of patients with Type 2 diabetes following the introduction of a diabetes shared care service. Diabet Med 2003;20:853-7.

25. Vinter-Repalust N, Petricek G, Katić M. Obstacles which patients with type 2 diabetes meet while adhering to the therapeutic regimen in everyday life: qualitative study. Croat Med J 2004;45:630-6.

26. Anthony $\mathrm{H}$, Valinsky L, Inbar Z, et al. Perceptions of hypertension treatment among patients with and without diabetes. BMC Fam Pract 2012;13:1.

27. Péres DS, Santos MA, Zanetti ML, et al. Difficulties of diabetic patients in the illness control: feelings and behaviors. Rev Lat Am Enfermagem 2007;15:1105-12.

28. Khatib R, Hall AS, Marshall K, et al. Exploring beliefs about medicines among adherent and non-adherent patients with coronary artery disease to establish medicines partnership. Eur Heart $J$ 2013;34:P2515.

29. Jolles EP, Padwal RS, Clark AM, et al. A qualitative study of patient perspectives about hypertension. ISRN Hypertension 2013;2013:1-10.

30. Kiawi E, Edwards R, Shu J, et al. Knowledge, attitudes, and behavior relating to diabetes and its main risk factors among urban residents in Cameroon: a qualitative survey. Ethn Dis 2006;16:503-9.

31. Al-Qazaz HK, Hassali MA, Shafie AA, et al. Perception and knowledge of patients with type 2 diabetes in Malaysia about their disease and medication: a qualitative study. Res Social Adm Pharm 2011;7:180-91.

32. Ali SM, Jusoff K. Barriers to optimal control of type 2 diabetes in Malaysian malay patients. Glob J Health Sci 2009;1:106.

33. Gascón JJ, Sánchez-Ortuño M, Llor B, et al. Why hypertensive patients do not comply with the treatment: results from a qualitative study. Fam Pract 2004;21:125-30.

34. Lai WA, Lew-Ting CY, Chie WC. How diabetic patients think about and manage their illness in Taiwan. Diabet Med 2005;22:286-92.

35. Peel E, Parry O, Douglas M, et al. Diagnosis of type 2 diabetes: a qualitative analysis of patients' emotional reactions and views about information provision. Patient Educ Couns 2004;53:269-75.

36. Marshall MN. Sampling for qualitative research. Fam Pract 1996;13:522-6.

37. Mason J. Qualitative researching: Sage, 2002.

38. Patton MQ. Qualitative evaluation and research methods: SAGE Publications,inc, 1990.

39. Sangasubana N. How to conduct ethnographic research. The qualitative report. 2011;16:567-73.

40. Kvale S. Doing interviews. Sage, 2008.

41. Boyatzis RE. Transforming qualitative information: thematic analysis and code development: Sage, 1998.

42. Al-Yousuf M, Akerele TM, Al-Mazrou YY. Organization of the Saudi health system. East Mediterr Health J 2002;8:499-500.

43. Alhusaini HA. Obstacles to the efficiency and performance of Saudi nurses at the Ministry of Health, Riyadh Region: analytical field study. Riyadh, Saudi Arabia: Ministry of Health, 2006. 